\title{
The impact of periodontal treatment on inflammatory markers and cellular parameters associated with atherosclerosis in patients after myocardial infarction
}

\author{
BARTŁOMIEJ GÓRSKI, RENATA GÓRSKA
}

Department of Periodontology and Oral Mucosa Diseases, Medical University of Warsaw, Warsaw, Poland

\begin{abstract}
Introduction: The aim of this study was to analyze whether periodontal treatment affects the cardiovascular risk profile of patients after myocardial infarction (MI).

Material and methods: The study included 30 patients with chronic periodontitis (ChP). Sociodemographic and medical variables were collected. Patients were provided with scaling and root planing (SRP) 3 months after MI ( $1^{\text {st }}$ visit). Periodontal examination and blood tests were performed immediately before SRP, then 1 month and 6 months after treatment ( $2^{\text {nd }}$ and $3^{\text {rd }}$ visit, respectively).

Results: A statistically significant decrease in blood hsCRP concentration and a decrease in the number of white blood cells $(W B C)$ and neutrophils between the first and the second visit were observed. At 6 months after SRP, the mean platelet volume (MPV) had increased with respect to the value at 1 month after treatment. Multivariate analysis showed that the associations between: 1) change in $L D L-C$ concentration and change in approximal plaque index value $(b=-0.546, p=0.005) ; 2)$ change in the number of monocytes and change in the plaque index value $(b=0.616, p=0.01) ; 3)$ change in MPV and change in probing pocket depth $(b=0.567, p=0.018)$ are all independent of the classic cardiovascular risk factors.

Conclusions: The obtained results indicate the existence of a relationship between the state of periodontal tissues on one hand and mediators of atherosclerosis and the number of immunologically competent cells on the other hand.
\end{abstract}

Key words: cardiovascular diseases, chronic periodontitis, $h s-C R P$.

(Centr Eur J Immunol 2018; 43 (4): 442-452)

\section{Introduction}

Cardiovascular diseases (CVD) constitute the most common cause of deaths and are associated with the death of 17.1 million people in the world annually [1]. In contemporary society we can observe a continuous increase in the prevalence of classic CVD risk factors such as smoking and passive smoking, hypertension, overweight, obesity and excessive consumption of sodium, which translates into steadily increasing statistics of cardiovascular events [2]. However, the coexistence of classic CVD risk factors does not fully explain the total cardiovascular risk in a given patient [3]. Recently, the importance of inflammatory and immunological processes has been underlined in the context of their impact on the development of atherosclerotic plaque [4].

Without doubt one of the most common inflammatory processes in society is chronic periodontitis. The occur- rence of advanced forms of chronic periodontitis in the population is estimated at 10-15\% [5]. Among adult Poles over 65 years of age, more than $50 \%$ of individuals have moderate or advanced periodontitis [6]. Much attention has been paid to the influence of periodontitis on CVD [7]. Research studies have shown that relative risk estimates for developing CVD in patients with periodontitis range from 1.24 to 1.34 [8]. This risk increases to 1.44 in people under 65 [9].

There are several mechanisms that can associate periodontitis with CVD $[8,10]$. In patients with chronic moderate and advanced periodontitis, the systemic inflammatory response increases. Treatment of periodontal disease leads to reduction of local inflammatory processes and to improvement of systemic biochemical parameters related to atherosclerosis, especially among patients suffering from CVD and/or diabetes [11]. Another link seems to be

Correspondence: Bartłomiej Górski, PhD, Department of Periodontology and Oral Mucosa Diseases, Medical University of Warsaw, 18 Miodowa St., 00-246 Warsaw, Poland, e-mail: bartek_g3@tlen.pl Submitted: 6.04.2018; Accepted: 23.04.2018 
periopathogens, which can penetrate into the bloodstream through damaged endothelium of capillaries. The concomitant periodontal inflamed surface area (PISA) in patients with chronic advanced periodontitis is $39 \mathrm{~cm}^{2}$ in size [12]. Among the microbiota, the greatest role is attributed to A. actinomycetemcomitans, $P$. gingivalis, $T$. forsythia and $P$. intermedia [13]. The mentioned periopathogens, whose presence in atherosclerotic plaque was confirmed by polymerase chain reaction, appear to induce thrombocyte activation and aggregation through collagen-like platelet aggregation-associated protein expression, which may be one of the first stages of thrombosis, eventually leading to an acute coronary episode [14]. Moreover, periopathogens - through innate immune mechanisms based on cellular responses - provoke and sustain chronic inflammatory responses ongoing at remote sites [15].

Among the modifiable factors and markers of cardiovascular risk, there are lipid disorders (increased concentrations of triglycerides (TG), total cholesterol (TC), and low-density lipoprotein cholesterol (LDL-C), and decreased concentrations of high-density lipoprotein cholesterol (HDL-C), mediators of inflammatory reaction (C-reactive protein [CRP]) and markers of fibrinolysis (fibrinogen). Only a few studies have evaluated associations between the state of periodontal tissues and the number of immunologically competent cells in blood. In our previously published study we reported the association between dental status and systemic lipid profile and inflammatory mediators in patients after myocardial infarction (MI) [16]. The results of multivariate analysis showed a relationship between LDL-C concentration, fibrinogen concentration and white blood cell count (WBC) on one hand and periodontitis and the number of lost teeth on the other hand.

The aim of the study was to assess the relationship between the state of periodontal tissues and the biochemical and cellular parameters associated with the development of atherosclerosis in patients with $\mathrm{ChP}$ after $\mathrm{MI}$ as well as verification of the impact of non-surgical periodontal treatment on the change in inflammatory mediators and the number of selected blood cell elements.

\section{Material and methods}

\section{Patient recruitment}

Subjects for the study were recruited from the pool of patients at the First Clinic and Department of Cardiology of Medical University of Warsaw hospitalized due to a recent acute MI on the second or third day after transfer from the Intensive Cardiac Care Unit to the General Cardiology Ward. All participants of the study were Caucasian Poles. The study group included 30 patients ( 6 women, 24 men), mean age 53.44 years $( \pm 7.65)$. The conditions for inclusion in the study were: 1) history of MI; 2) age under 65 years; 3 ) presence of at least 8 teeth (except for third molars); 4) diagnosed chronic periodontitis. MI (STEMI and NSTEMI) was diagnosed in line with the Guidelines of ESC. The following exclusion criteria were applied: 1) neoplastic disease; 2) rheumatic disease; 3 ) autoimmune disease; 4) chronic liver diseases; 5) chronic kidney disease at $4^{\text {th }}$ and $5^{\text {th }}$ stages; 6) stroke.

The study was conducted observing ethical norms resulting from the Helsinki Declaration of 1975, as revised in 2000. The permission of the Bioethics Committee at the Medical University of Warsaw (approval number KB145/2011) was obtained for conducting the research. The subjects in the study expressed their informed consent to participate in the project by signing an informed consent form. Social and general medical history of participants was taken. They were also subjected to physical, dental and periodontal examinations.

\section{Patient population}

In order to carry out the study, a properly constructed questionnaire was used. Based on information collected from the interview, data on age, income, education, atherosclerotic disease in the family and nicotinism were obtained. Income was determined on the basis of income per family member per month: < 800 PLN, 800-1500 PLN, $>1500$ PLN. Education was defined as primary, secondary and university. Study participants were categorized as current smokers if they reported smoking at least 10 cigarettes per day continually for at least 5 years, past smokers if they reported smoking in the past and subjects who never smoked cigarettes (never smokers).

Hypertension was defined as systolic blood pressure $(\mathrm{SBP}) \geq 140 \mathrm{~mm} \mathrm{Hg}$ or diastolic blood pressure (DBP) $\geq 90 \mathrm{~mm} \mathrm{Hg}$ in three consecutive measurements performed at five-minute intervals or if the patient was taking antihypertensive drugs. In the survey, the mean value of SBP and DBP obtained on the limb with a higher mean pressure was noted.

Diabetes was diagnosed if fasting blood glucose concentration was above $126 \mathrm{mg} / \mathrm{dl}$ or if the patient was taking appropriate medications

Measurement of height (in $\mathrm{cm}$ ) and body weight (in $\mathrm{kg}$ ) was carried out using a medical scale with an increase meter. Body height measurement was taken in a standing position with an accuracy of $1 \mathrm{~cm}$. Body mass measurement was taken with an accuracy of $0.1 \mathrm{~kg}$. Body mass index (BMI) was calculated by dividing body weight (in $\mathrm{kg}$ ) by height (in $\mathrm{m}^{2}$ ). BMI $25-29.9 \mathrm{~kg} / \mathrm{m}^{2}$ was defined as overweight, and BMI $\geq 30 \mathrm{~kg} / \mathrm{m}^{2}$ as obesity.

Dental examination determined the number of teeth present in the oral cavity and the number of extracted teeth (except for the third molars). Periodontal examination was carried out at 4 sites around all teeth: mesio-buccal (MB), buccal (B), disto-buccal (DB) and lingual (L). Only probing pocket depth (PPD) and clinical attachment level (CAL) were determined in order to make a preliminary diagnosis of chronic periodontitis [17]. 
Patients who met all the inclusion criteria and agreed to be included in the study program were invited for another visit to the Department of Periodontology and Oral Mucosa Diseases of the Medical University of Warsaw. The visit took place three months after the end of hospitalization.

\section{Laboratory analyses, periodontal measurements and treatment}

Blood was collected from fasting participants and immediately transferred to the Central Laboratory in order to determine concentrations of serum TC (norm $\leq 190 \mathrm{mg} / \mathrm{dl}$ ), TG (norm $\leq 150 \mathrm{mg} / \mathrm{dl})$, LDL-C (norm $\leq 115 \mathrm{mg} / \mathrm{dl})$, HDL-C (male norm $>40 \mathrm{mg} / \mathrm{dl}$, female norm $>46 \mathrm{mg} / \mathrm{dl}$ ), hsCRP (reference value: $0-5 \mathrm{mg} / \mathrm{l}$ ), fibrinogen (reference value: $200-400 \mathrm{mg} / \mathrm{dl}$ ), leucocytes (WBC) (norm 4.0-11.0 $10^{3} / \mu \mathrm{l}$ ), neutrophil counts (norm 1.8-7.7 103/ $\mathrm{ll}$ ), lymphocyte counts (norm 1.0-4.5 103/ $/ \mathrm{l}$ ), monocyte count (norm 0-0.08 $10^{3} / \mu \mathrm{l}$ ), platelet count (PLT) (standard 130-450 103/ $/ \mathrm{l}$ ) and mean platelet volume (MPV) (9.0-13.0 fl).

The periodontal examination was carried out by a calibrated researcher. The study was conducted in artificial lighting, using a dental mirror and a periodontal probe calibrated every $1 \mathrm{~mm}$ (Hu-Friedy PCPUNC 15). The study did not include third molars. The dichotomous plaque index (PI) according to O'Leary was determined on four surfaces of all teeth (mesial, distal, lingual and buccal). The index was evaluated as the ratio of plaque surface area to all examined surfaces [18]. In order to assess the effectiveness of interdental space cleaning, the dichotomous approximal plaque index on contact surfaces (API) according to Lange was evaluated as the ratio between interdental spaces with plaque to all interdental spaces [19]. The study also evaluated bleeding on probing $(\mathrm{BoP})$ according to Ainamo and Bay, and the exam-

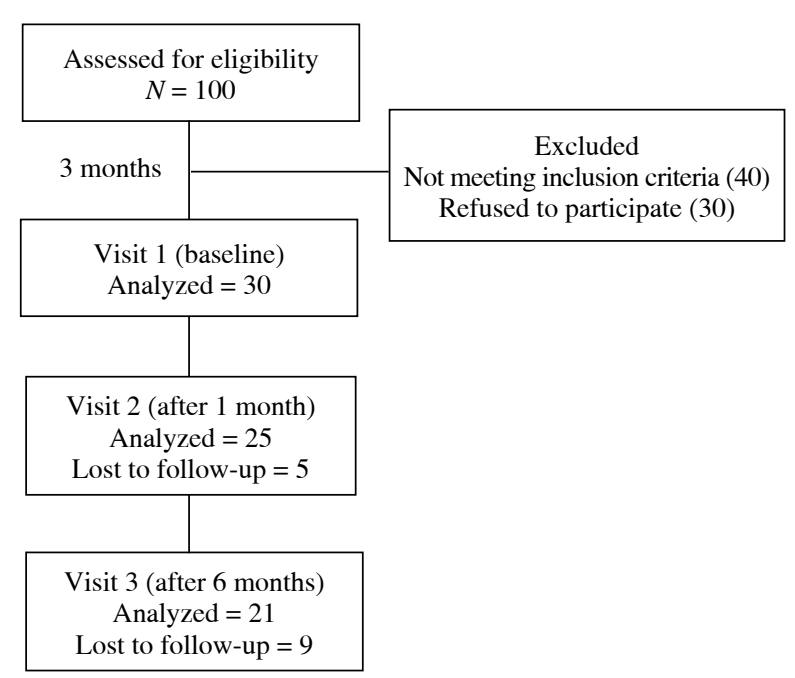

Fig. 1. Study summary ination was carried out at 4 points around all teeth: MB, B, DB and L. BoP was calculated by dividing the number of bleeding points by the number of all examined points [20]. PPD and clinical attachment level (CAL) were assessed at 4 points around all teeth (MB, B, DB, L). PPD was defined as the distance from the gingival margin to the bottom of the pocket as measured by probing (in $\mathrm{mm}$ ). CAL was defined as the distance between the bottom of the pocket determined by probing and the cementoenamel junction (in $\mathrm{mm}$ ). The measurements were rounded down to full $\mathrm{mm}$. The number of active (bleeding) periodontal pockets with a depth of $\geq 4 \mathrm{~mm}$ was also verified.

Precise diagnosis of periodontitis was made in accordance with the case definitions proposed by the Centers for Disease Control and AAP (Page and Eke definition) as follows:

- mild periodontitis as $\geq 2$ interproximal sites with CAL $\geq 3 \mathrm{~mm}$ and $\geq 2$ interproximal sites with PD $\geq 4 \mathrm{~mm}$ (not on the same tooth),

- moderate periodontitis as $\geq 2$ interproximal sites with CAL $\geq 4 \mathrm{~mm}$ or $\geq 2$ interproximal sites with PPD $\geq 5 \mathrm{~mm}$ (not on the same tooth),

- severe periodontitis as $\geq 2$ interproximal sites with $\mathrm{CAL} \geq 6 \mathrm{~mm}$ (not on the same tooth) and $\geq 1$ interproximal site with PPD $\geq 5 \mathrm{~mm}$ [17].

After a thorough periodontal examination, all patients underwent classic non-surgical treatment (scaling and root planing - SRP) within all quadrants using hand and ultrasonic instruments with addition of $0.2 \%$ chlorhexidine solution to the coolant. Subsequently, tooth surfaces were polished using rubber bands and polishing paste. Then the patients were given individual oral hygiene instructions that included plaque control in interdental spaces. Patients were motivated to properly clean all dental surfaces. Additionally, for 2 weeks after SRP, patients were recommended to use a $0.1 \%$ chlorhexidine mouthwash.

\section{Follow-up visits}

Subsequent visits took place at 1 month and 6 months after SRP (Fig. 1). During each visit, blood samples were taken from the patients on an empty stomach to perform determinations of the previously mentioned biochemical and cellular parameters. A full periodontal examination was carried out according to the previously described protocol. If necessary, teeth were cleaned of soft and hard deposits by means of repeated SRP.

\section{Statistical analysis}

Descriptive statistics such as means, standard deviations (SD) as well confidence intervals (CI) at 95\% confidence level were calculated for dental and biochemical parameters. Comparisons of means of dental and biochemical characteristics between subsequent measurements were conducted using the $t$-test for paired data. 
Relationships between changes (differences between subsequent measurements) in biochemical variables and periodontal parameters were evaluated using Pearson's correlation coefficient and multiple linear regression. In multiple regression analysis changes in periodontal variables were considered as independent variables, while changes in biochemical parameters were considered as dependent variables. In the first stage, gender and age of the patients were included as additional independent variables (Model I). In the second stage, other CVD risk factors, such as tobacco smoking, arterial hypertension, diabetes mellitus, BMI, atherosclerosis in the family, income and education, were added (Model II). Statistical significance for all analyses was set at the 0.05 probability level. The analyses were conducted using Statistica 13 software.

\section{Results}

Table 1 presents the demographic and general-medical characteristics of the study group. Smoking was reported by 22 participants (73\%). Twelve individuals $(40 \%)$ suffered from hypertension, $4(13 \%)$ from diabetes. Over $80 \%$ of subjects (24 patients) demonstrated anomalous BMI values.

Table 2 includes assessed periodontal indices and parameters as well as changes in the scope of these variables at defined time points. Patients' oral hygiene was unsatisfactory, but a downward trend in PI and API was observed. Subjects had an average of 19 teeth. Moderate ChP was diagnosed in 12 subjects $(40 \%)$ and the advanced form in 15 persons $(50 \%)$.

Table 3 presents values of TC, TG, LDL-C, HDL-C, hsCRP and fibrinogen concentrations in blood as well as the number of assessed morphotic parameters before periodontal treatment, 1 month after and 6 months after SRP. A statistically significant reduction was observed in blood hsCRP concentration between the first and the second visit as well as a reduction in the number of WBC and neutrophils. Six months after the end of periodontal treatment, MPV increased with respect to the value after 1 month of treatment.

On the basis of univariate analysis (Tables 4 and 5), a correlation between changes in selected biochemical parameters and changes in PI, API, PPD and CAL was observed. Multivariate analysis which was corrected in the first stage for gender and age (Table 6), and then for the other assessed CVD risk factors, showed that the relationships between: 1) change in LDL-C concentration between the $2^{\text {nd }}$ and the $1^{\text {st }}$ visit correlated with the change in the API value; 2) change in the number of monocytes between the $2^{\text {nd }}$ and the $1^{\text {st }}$ visit correlated with the change in the PI value; 3) MPV change between the $3^{\text {rd }}$ and $2^{\text {nd }}$ visit correlated with PPD change are all independent of age, gender, income, ed- ucation, atherosclerosis in the family, smoking, hypertension, diabetes and body weight disorders.

\section{Discussion}

Periodontal diseases and CVD have many common risk factors, such as higher prevalence among older men, smokers, with diabetes, body weight disorders, dyslipidemia and lower socioeconomic status, some of which are modifiable and some are non-modifiable. In our own study, frequent nicotinism, hypertension, overweight and obesity in patients after MI was observed. The extensive INTER-HEART study showed that dyslipidemia yields a $49 \%$ risk of MI [3]. In the case of patients with stable $\mathrm{CHD}$, raised CRP concentration increases the risk of MI,

Table 1. Demographic and medical characteristics of the study group $(N=30)$

\begin{tabular}{|c|c|}
\hline Demographic and medical variables & \\
\hline \multicolumn{2}{|l|}{ Gender, $n(\%)$} \\
\hline Women & $6(20)$ \\
\hline Men & $24(80)$ \\
\hline Age, mean \pm SD & $53.44 \pm 7.65$ \\
\hline \multicolumn{2}{|l|}{ Income, $n(\%)$} \\
\hline$>1500$ PLN & $9(30)$ \\
\hline 800-1500 PLN & $14(46.67)$ \\
\hline$<800$ PLN & $7(23.33)$ \\
\hline \multicolumn{2}{|l|}{ Education, $n(\%)$} \\
\hline Higher & $4(13.33)$ \\
\hline Secondary & $21(70)$ \\
\hline Primary & $5(16.67)$ \\
\hline Atherosclerosis in family, $n(\%)$ & $4(13.3)$ \\
\hline \multicolumn{2}{|l|}{ Smoking habit, $n(\%)$} \\
\hline Never & $3(10)$ \\
\hline Past & $5(16.67)$ \\
\hline Current & $22(73.33)$ \\
\hline Arterial hypertension, $n(\%)$ & $12(40)$ \\
\hline Systolic blood pressure $(\mathrm{mm} \mathrm{Hg})$, mean $\pm \mathrm{SD}$ & $135.2 \pm 22.7$ \\
\hline Diastolic blood pressure $(\mathrm{mm} \mathrm{Hg})$, mean $\pm \mathrm{SD}$ & $80.3 \pm 14.1$ \\
\hline Diabetes mellitus, $n(\%)$ & $4(13.34)$ \\
\hline $\mathrm{BMI}\left(\mathrm{kg} / \mathrm{m}^{2}\right)$, mean $\pm \mathrm{SD}$ & $28.92 \pm 4.63$ \\
\hline \multicolumn{2}{|l|}{ Body weight, $n(\%)$} \\
\hline Normal & $6(20)$ \\
\hline Overweight & $13(43.33)$ \\
\hline Obesity & $11(36.67)$ \\
\hline
\end{tabular}

$S D$ - standard deviation, $n$ - number, BMI - body mass index 
Table 2. Dental characteristics of the study group $(N=30)$

\begin{tabular}{|c|c|c|c|c|c|}
\hline Variable & Baseline & 1 month & 6 months & $\begin{array}{l}\text { P1 (baseline } \\
-1 \text { month) }\end{array}$ & $\begin{array}{c}P 2 \\
\text { (1-6 months) }\end{array}$ \\
\hline $\begin{array}{l}\text { Plaque index }(\mathrm{PI})(\%) \text {, mean } \\
(95 \% \mathrm{CI}) \pm \mathrm{SD}\end{array}$ & $\begin{array}{c}86.11(79.16-93.06) \\
\pm 18.6\end{array}$ & $\begin{array}{c}69.56(59.49-79.63) \\
\pm 24.4\end{array}$ & $\begin{array}{c}80.01(66.81-93.20) \\
\pm 22.85\end{array}$ & $0.001^{*}$ & 0.324 \\
\hline $\begin{array}{l}\text { Approximal plaque index (API) } \\
(\%), \text { mean }(95 \% \mathrm{CI}) \pm \mathrm{SD}\end{array}$ & $\begin{array}{l}97.61(94.59-100) \\
\pm 8.08\end{array}$ & $\begin{aligned} 89.57 & (81.75-97.39) \\
& \pm 18.94\end{aligned}$ & $\begin{array}{l}86.91(69.73-100) \\
\pm 29.76\end{array}$ & $0.043^{*}$ & 0.734 \\
\hline $\begin{array}{l}\text { Bleeding on probing index }(\mathrm{BoP}) \\
(\%), \text { mean }(95 \% \mathrm{CI}) \pm \mathrm{SD}\end{array}$ & $\begin{array}{c}37.0(28.11-45.89) \\
\pm 23.81\end{array}$ & $\begin{aligned} 35.11 & (26.71-43.52) \\
& \pm 20.36\end{aligned}$ & $\begin{array}{c}45.3(32.50-58.09) \\
\quad \pm 22.16\end{array}$ & 0.850 & $0.019^{*}$ \\
\hline $\begin{array}{l}\text { Probing pocket depth }(\mathrm{PPD})(\mathrm{mm}) \text {, } \\
\text { mean }(95 \% \mathrm{CI}) \pm \mathrm{SD}\end{array}$ & $2.99(2.42-3.57) \pm 1.53$ & $\begin{array}{c}2.71(2.36-3.06) \\
\pm 0.85\end{array}$ & $\begin{array}{c}2.84(1.99-3.68) \\
\quad \pm 1.47\end{array}$ & 0.815 & 0.766 \\
\hline $\begin{array}{l}\text { Bleeding periodontal pockets (bPP) } \\
(\%) \text {, mean }(95 \% \mathrm{CI}) \pm \mathrm{SD}\end{array}$ & $\begin{array}{c}21.92(15.06-28.77) \\
\pm 18.35\end{array}$ & $\begin{aligned} 18.10 & (10.78-25.42) \\
& \pm 17.73\end{aligned}$ & $\begin{array}{c}24.12(9.74-38.5) \\
\quad \pm 24.91\end{array}$ & 0.069 & 0.770 \\
\hline $\begin{array}{l}\text { Clinical attachment level (CAL) } \\
(\mathrm{mm}), \text { mean }(95 \% \mathrm{CI}) \pm \mathrm{SD}\end{array}$ & $\begin{array}{c}3.85(3.28-4.41) \\
\quad \pm 1.5\end{array}$ & $\begin{array}{c}3.64(3.03-4.24) \\
\quad \pm 1.47\end{array}$ & $\begin{array}{c}4.41(3.33-5.5) \\
\quad \pm 1.88\end{array}$ & $0.023^{*}$ & 0.382 \\
\hline \multicolumn{6}{|l|}{ Periodontal status, $n(\%)$} \\
\hline Mild periodontitis & $3(10)$ & - & - & & \\
\hline Moderate periodontitis & $12(40)$ & - & - & & \\
\hline Severe periodontitis & $15(50)$ & - & - & & \\
\hline Number of teeth, median (Q1-Q3), $n$ & $19(15-24)$ & - & - & - & - \\
\hline
\end{tabular}

Table 3. Biochemical characteristics of the study group $(N=30)$

\begin{tabular}{|c|c|c|c|c|c|}
\hline Variables & Baseline & 1 month & 6 months & $\begin{array}{l}\text { P1 (baseline } \\
-1 \text { month) }\end{array}$ & $\begin{array}{l}\text { P2 (1-6 } \\
\text { months }\end{array}$ \\
\hline $\begin{array}{l}\mathrm{TC}, \text { mean }(95 \% \mathrm{CI}) \\
\pm \mathrm{SD}(\mathrm{mg} / \mathrm{dl})\end{array}$ & $\begin{array}{c}158.38(144.79-171.97) \\
\pm 36.39\end{array}$ & $\begin{array}{c}154.96(136.735-173.57) \\
\pm 44.07\end{array}$ & $\begin{array}{c}159.05(143.08-175.02) \\
\pm 34.12\end{array}$ & 0.446 & 0.534 \\
\hline $\begin{array}{l}\mathrm{TG}, \text { mean }(95 \% \mathrm{CI}) \\
\pm \mathrm{SD}(\mathrm{mg} / \mathrm{dl})\end{array}$ & $\begin{array}{c}154.07(119.51-188.63) \\
\pm 90.86\end{array}$ & $\begin{array}{c}155.25(122.39-194.11) \\
\pm 84.91\end{array}$ & $\begin{array}{c}153.55(119.8-187.30) \\
\pm 72.11\end{array}$ & 0.854 & 0.505 \\
\hline $\begin{array}{l}\text { LDL-C, mean }(95 \% \mathrm{CI}) \\
\pm \mathrm{SD}(\mathrm{mg} / \mathrm{dl})\end{array}$ & $\begin{array}{c}82.62(69.36-95.87) \\
\pm 32.82\end{array}$ & $\begin{array}{c}71.71(60.88-82.55) \\
\pm 23.8\end{array}$ & $\begin{array}{c}85.65(68.03-103.26) \\
\pm 34.26\end{array}$ & 0.078 & 0.695 \\
\hline $\begin{array}{l}\text { HDL-C, mean }(95 \% \mathrm{CI}) \\
\pm \mathrm{SD}(\mathrm{mg} / \mathrm{dl})\end{array}$ & $\begin{array}{c}47.06(43.32-50.80) \\
\pm 9.84\end{array}$ & $\begin{array}{c}49.58(45.73-53.44) \\
\pm 9.13\end{array}$ & $\begin{array}{c}47.6(42.71-52.49) \\
\quad \pm 10.44\end{array}$ & 0.270 & 0.203 \\
\hline $\begin{array}{l}\text { hsCRP, mean }(95 \% \mathrm{CI}) \\
\pm \mathrm{SD}(\mathrm{mg} / \mathrm{l})\end{array}$ & $\begin{array}{c}2.97(2.24-3.69) \\
\pm 1.9\end{array}$ & $\begin{array}{c}2.09(1.5-2.69) \\
\pm 1.41\end{array}$ & $\begin{array}{c}2.51(1.03-3.98) \\
\pm 3.16\end{array}$ & $0.033^{*}$ & 0.536 \\
\hline $\begin{array}{l}\text { Fibrinogen, mean }(95 \% \mathrm{CI}) \\
\pm \mathrm{SD}(\mathrm{mg} / \mathrm{dl})\end{array}$ & $\begin{array}{c}318.03(300.3-335.77) \\
\pm 46.62\end{array}$ & $\begin{array}{c}307.21(285.2-329.21) \\
\pm 52.611\end{array}$ & $\begin{aligned} 296.19 & (256.88-335.5) \\
& \pm 86.36\end{aligned}$ & 0.534 & 0.618 \\
\hline $\begin{array}{l}\text { WBC, mean }(95 \% \mathrm{CI}) \\
\pm \mathrm{SD}(103 \mu \mathrm{l})\end{array}$ & $\begin{array}{c}7.39 \text { (6.63-8.15) } \\
\pm 2.01\end{array}$ & $\begin{array}{c}6.78(6.11-7.45) \\
\quad \pm 1.62\end{array}$ & $\begin{array}{c}7.07 \text { (6.06-8.07) } \\
\pm 2.21\end{array}$ & $0.021^{*}$ & 0.055 \\
\hline $\begin{array}{l}\text { NEUT, mean }(95 \% \mathrm{CI}) \\
\pm \mathrm{SD}(103 \mu \mathrm{l})\end{array}$ & $\begin{array}{c}4.55(3.96-5.14) \\
\pm 1.52\end{array}$ & $\begin{array}{c}3.97(3.44-4.50) \\
\quad \pm 1.28\end{array}$ & $\begin{array}{c}3.99 \text { (3.26-4.73) } \\
\quad \pm 1.43\end{array}$ & $0.015^{*}$ & 0.276 \\
\hline $\begin{array}{l}\text { LYMPH, mean }(95 \% \mathrm{CI}) \\
\pm \mathrm{SD}(103 \mu \mathrm{l})\end{array}$ & $\begin{array}{c}2.06(1.84-2.28) \\
\pm 0.57\end{array}$ & $\begin{array}{c}1.96(1.73-2.2) \\
\pm 0.57\end{array}$ & $\begin{array}{c}1.77(1.51-2.03) \\
\pm 0.5\end{array}$ & 0.050 & 0.458 \\
\hline $\begin{array}{l}\text { MONO, mean }(95 \% \mathrm{CI}) \\
\pm \mathrm{SD}(103 \mu \mathrm{l})\end{array}$ & $\begin{array}{c}0.56(0.49-0.62) \\
\pm 0.17\end{array}$ & $\begin{array}{c}0.79(0.23-1.36) \\
\pm 1.36\end{array}$ & $\begin{array}{c}0.48(0.39-0.58) \\
\pm 0.18\end{array}$ & 0.418 & 0.531 \\
\hline $\begin{array}{l}\text { PLT, mean }(95 \% \mathrm{CI}) \\
\pm \mathrm{SD}(103 \mu \mathrm{l})\end{array}$ & $\begin{array}{c}224.9(203.92-245.87) \\
\pm 55.15\end{array}$ & $\begin{array}{c}213.92(188.23-239.61) \\
\pm 62.22\end{array}$ & $\begin{array}{c}216.45(176.82-256.08) \\
\pm 82.22\end{array}$ & 0.184 & 0.074 \\
\hline $\begin{array}{l}\text { MPV, mean }(95 \% \mathrm{CI}) \\
\pm \mathrm{SD}(\mathrm{fl})\end{array}$ & $\begin{array}{c}9.48(9.02-9.95) \\
\pm 1.23\end{array}$ & $\begin{array}{c}9.19(8.76-9.62) \\
\pm 1.04\end{array}$ & $\begin{aligned} 10.79 & (9.75-11.82) \\
& \pm 2.28\end{aligned}$ & 0.087 & $0.021^{*}$ \\
\hline
\end{tabular}

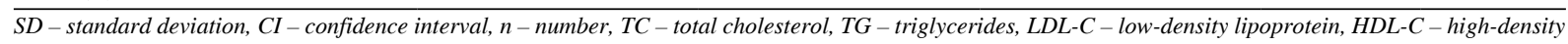
lipoprotein, hsCRP-high-sensitivity C-reactive protein, WBC-white blood cells, NEUT-neutrophils, LYMPH-lymphocytes, MONO-monocytes, PLT-platelets, $M P V-$ mean platelet volume, ${ }^{*}$ - statistical significance 
Table 4. Correlations between changes in biochemical parameters from baseline to 1 month and dental status

\begin{tabular}{|c|c|c|c|c|c|c|c|}
\hline & $\begin{array}{c}\text { Plaque index } \\
(\%)\end{array}$ & $\begin{array}{c}\text { Approximal } \\
\text { plaque index } \\
(\%)\end{array}$ & $\begin{array}{c}\text { Bleeding on } \\
\text { probing index } \\
(\%)\end{array}$ & $\begin{array}{c}\text { Probing } \\
\text { pocket depth } \\
(\mathbf{m m})\end{array}$ & $\begin{array}{c}\text { Bleeding } \\
\text { periodontal } \\
\text { pockets }(\%)\end{array}$ & $\begin{array}{c}\text { Clinical } \\
\text { attachment } \\
\text { level }(\mathbf{m m})\end{array}$ & $\begin{array}{l}\text { Number of } \\
\text { missing teeth }\end{array}$ \\
\hline $\mathrm{TC}(\mathrm{mg} / \mathrm{dl})$ & $\begin{aligned} r & =-0.13 \\
p & =0.533\end{aligned}$ & $\begin{aligned} r & =-0.14 \\
p & =0.520\end{aligned}$ & $\begin{aligned} r & =-0.21 \\
p & =0.316\end{aligned}$ & $\begin{array}{c}r=0.35 \\
p=0.091\end{array}$ & $\begin{array}{c}r=0.39 \\
p=0.058\end{array}$ & $\begin{array}{c}r=0.12 \\
p=0.568\end{array}$ & $\begin{array}{c}r=0.15 \\
p=0.485\end{array}$ \\
\hline $\mathrm{TG}(\mathrm{mg} / \mathrm{dl})$ & $\begin{array}{l}r=-0.11 \\
p=0.616\end{array}$ & $\begin{array}{c}r=0.01 \\
p=0.965\end{array}$ & $\begin{array}{l}r=-0.08 \\
p=0.713\end{array}$ & $\begin{array}{c}r=0.15 \\
p=0.491\end{array}$ & $\begin{array}{c}r=0.18 \\
p=0.393\end{array}$ & $\begin{array}{l}r=-0.01 \\
p=0.963\end{array}$ & $\begin{array}{c}r=0.07 \\
p=0.755\end{array}$ \\
\hline LDL-C (mg/dl) & $\begin{array}{l}r=-0.26 \\
p=0.282\end{array}$ & $\begin{aligned} r & =-0.46 \\
p & =0.049^{*}\end{aligned}$ & $\begin{array}{l}r=-0.13 \\
p=0.596\end{array}$ & $\begin{aligned} r & =-0.05 \\
p & =0.831\end{aligned}$ & $\begin{array}{c}r=0.04 \\
p=0.873\end{array}$ & $\begin{aligned} r & =-0.10 \\
p & =0.687\end{aligned}$ & $\begin{array}{l}r=-0.08 \\
p=0.754\end{array}$ \\
\hline HDL-C (mg/dl) & $\begin{aligned} r & =0.30 \\
p & =0.157\end{aligned}$ & $\begin{array}{c}r=0.17 \\
p=0.421\end{array}$ & $\begin{aligned} r & =-0.03 \\
p & =0.905\end{aligned}$ & $\begin{array}{c}r=0.60 \\
p=0.002^{*}\end{array}$ & $\begin{array}{c}r=0.43 \\
p=0.035^{*}\end{array}$ & $\begin{array}{c}r=0.34 \\
p=0.101\end{array}$ & $\begin{aligned} r & =-0.03 \\
p & =0.897\end{aligned}$ \\
\hline hsCRP (mg/l) & $\begin{array}{l}r=-0.33 \\
p=0.120\end{array}$ & $\begin{aligned} r & =-0.17 \\
p & =0.434\end{aligned}$ & $\begin{aligned} r & =-0.28 \\
p & =0.184\end{aligned}$ & $\begin{array}{c}r=0.14 \\
p=0.509\end{array}$ & $\begin{array}{c}r=0.04 \\
p=0.858\end{array}$ & $\begin{array}{c}r=0.26 \\
p=0.225\end{array}$ & $\begin{array}{c}r=0.05 \\
p=0.812\end{array}$ \\
\hline Fibrinogen (mg/dl) & $\begin{array}{c}r=0.19 \\
p=0.376\end{array}$ & $\begin{array}{c}r=0.24 \\
p=0.251\end{array}$ & $\begin{aligned} r & =-0.38 \\
p & =0.067\end{aligned}$ & $\begin{aligned} r & =-0.24 \\
p & =0.260\end{aligned}$ & $\begin{array}{l}r=-0.12 \\
p=0.564\end{array}$ & $\begin{aligned} r & =0.09 \\
p & =0.676\end{aligned}$ & $\begin{array}{c}r=0.16 \\
p=0.463\end{array}$ \\
\hline WBC $\left(10^{3} / \mu \mathrm{l}\right)$ & $\begin{array}{c}r=0.14 \\
p=0.516\end{array}$ & $\begin{array}{c}r=0.17 \\
p=0.420\end{array}$ & $\begin{aligned} r & =-0.08 \\
p & =0.710\end{aligned}$ & $\begin{array}{c}r=0.05 \\
p=0.820\end{array}$ & $\begin{array}{c}r=0.12 \\
p=0.583\end{array}$ & $\begin{array}{c}r=0.04 \\
p=0.869\end{array}$ & $\begin{array}{c}r=0.20 \\
p=0.351\end{array}$ \\
\hline $\operatorname{NEUT}\left(10^{3} / \mu \mathrm{l}\right)$ & $\begin{array}{c}r=0.04 \\
p=0.861\end{array}$ & $\begin{aligned} r & =0.09 \\
p & =0.675\end{aligned}$ & $\begin{aligned} r & =0.00 \\
p & =0.997\end{aligned}$ & $\begin{aligned} r & =0.05 \\
p & =0.802\end{aligned}$ & $\begin{aligned} r & =0.10 \\
p & =0.655\end{aligned}$ & $\begin{aligned} r & =0.04 \\
p & =0.860\end{aligned}$ & $\begin{array}{c}r=0.12 \\
p=0.590\end{array}$ \\
\hline LYMPH $\left(10^{3} / \mu \mathrm{l}\right)$ & $\begin{array}{c}r=0.28 \\
p=0.193\end{array}$ & $\begin{array}{c}r=0.29 \\
p=0.170\end{array}$ & $\begin{array}{l}r=-0.14 \\
p=0.515\end{array}$ & $\begin{array}{c}r=0.13 \\
p=0.559\end{array}$ & $\begin{array}{c}r=0.15 \\
p=0.477\end{array}$ & $\begin{array}{c}r=0.10 \\
p=0.641\end{array}$ & $\begin{array}{c}r=0.30 \\
p=0.158\end{array}$ \\
\hline $\operatorname{MONO}\left(10^{3} / \mu \mathrm{l}\right)$ & $\begin{array}{c}r=0.41 \\
p=0.049^{*}\end{array}$ & $\begin{array}{c}r=0.38 \\
p=0.069\end{array}$ & $\begin{array}{c}r=0.07 \\
p=0.729\end{array}$ & $\begin{aligned} r & =-0.34 \\
p & =0.103\end{aligned}$ & $\begin{aligned} r & =-0.37 \\
p & =0.074\end{aligned}$ & $\begin{aligned} r & =-0.28 \\
p & =0.193\end{aligned}$ & $\begin{array}{c}r=0.04 \\
p=0.841\end{array}$ \\
\hline $\operatorname{PLT}\left(10^{3} / \mu \mathrm{l}\right)$ & $\begin{array}{l}r=-0.20 \\
p=0.349\end{array}$ & $\begin{aligned} r & =-0.14 \\
p & =0.526\end{aligned}$ & $\begin{array}{c}r=0.18 \\
p=0.390\end{array}$ & $\begin{aligned} r & =-0.23 \\
p & =0.279\end{aligned}$ & $\begin{array}{l}r=-0.34 \\
p=0.102\end{array}$ & $\begin{array}{l}r=-0.01 \\
p=0.962\end{array}$ & $\begin{array}{l}r=-0.13 \\
p=0.537\end{array}$ \\
\hline MPV (fl) & $\begin{array}{c}r=0.13 \\
p=0.537\end{array}$ & $\begin{array}{l}r=-0.02 \\
p=0.931\end{array}$ & $\begin{array}{l}r=-0.07 \\
p=0.757\end{array}$ & $\begin{array}{c}r=0.24 \\
p=0.266\end{array}$ & $\begin{array}{c}r=0.31 \\
p=0.145\end{array}$ & $\begin{array}{c}r=0.13 \\
p=0.559\end{array}$ & $\begin{array}{c}r=0.15 \\
p=0.476\end{array}$ \\
\hline
\end{tabular}

and in the case of patients with unstable CHD and history of MI, elevated CRP worsens the prognosis and increases the risk of complications. CRP concentration is an independent predictor of cardiovascular risk, even after considering classic risk factors such as hypertension and elevated TC level [21]. On the other hand, elevated level of fibrinogen correlates with atherosclerosis severity and patient mortality [21]. Exact mechanisms of the effect of periodontal treatment on the course of CVD remain unknown, but these processes may be related to both direct control and attenuating local inflammatory reactions (which can be expressed by changes in the BoP index), as well as to regulating the modifiable risk factors common for periodontal diseases and CVD [22].

In this study, $50 \%$ of patients were diagnosed with chronic advanced generalized periodontitis. The main etiological factor of periodontitis is periopathogens present in the bacterial biofilm; therefore an essential element of treatment is an improvement of mechanical home plaque control by the patient through regular brushing and cleaning of the interdental spaces. The main goal of the hygienic phase is to meet the requirement of oral health education
(OHE), that is a reduction of API below $25 \%$ and BoP below $10 \%$ [23]. Only after fulfilling this goal is it possible to implement the proper scheme of comprehensive periodontal treatment. The most important aspect of therapy is mechanical instrumentation with hand instruments or mechanical scalers [24]. These procedures are performed in order to remove bacterial biofilm together with mineralized deposits from the tooth surface and to smooth the root cement surface (SRP) in separate sections of dentition at specific time intervals. The treatment strategy based on simultaneous cleaning and preparation of all teeth is aimed at preventing any translocation of periopathogens from untreated sites to the pockets already subjected to non-surgical treatment. This procedure should be implemented in patients with generalized moderate and advanced $\mathrm{ChP}$ with a high risk of cross-contamination of pockets due to a large amount of supragingival deposits [25]. Therefore, in this study we decided to use the treatment protocol consisting of a single-visit instrumentation of all teeth. Patients were instructed on optimal oral hygiene, and treatment results were reassessed after one month and after 6 months. Oral hygiene of the subjects was unsatisfactory, but a down- 
Table 5. Correlations between changes in biochemical parameters from 1 month to 6 months after treatment and dental status

\begin{tabular}{|c|c|c|c|c|c|c|c|}
\hline & $\begin{array}{c}\text { Plaque index } \\
(\%)\end{array}$ & $\begin{array}{c}\text { Approximal } \\
\text { plaque index } \\
(\%)\end{array}$ & $\begin{array}{c}\text { Bleeding on } \\
\text { probing index } \\
(\%)\end{array}$ & $\begin{array}{c}\text { Probing } \\
\text { pocket depth } \\
(\mathbf{m m})\end{array}$ & $\begin{array}{c}\text { Bleeding } \\
\text { periodontal } \\
\text { pockets (\%) }\end{array}$ & $\begin{array}{c}\text { Clinical } \\
\text { attachment } \\
\text { level }(\mathbf{m m})\end{array}$ & $\begin{array}{l}\text { Number of } \\
\text { missing teeth }\end{array}$ \\
\hline $\mathrm{TC}(\mathrm{mg} / \mathrm{dl})$ & $\begin{aligned} r & =-0.01 \\
p & =0.974\end{aligned}$ & $\begin{aligned} r & =-0.11 \\
p & =0.723\end{aligned}$ & $\begin{array}{c}r=0.05 \\
p=0.859\end{array}$ & $\begin{array}{c}r=0.16 \\
p=0.606\end{array}$ & $\begin{array}{c}r=0.10 \\
p=0.748\end{array}$ & $\begin{array}{c}r=0.12 \\
p=0.687\end{array}$ & $\begin{aligned} r & =-0.18 \\
p & =0.491\end{aligned}$ \\
\hline $\mathrm{TG}(\mathrm{mg} / \mathrm{dl})$ & $\begin{aligned} r & =-0.26 \\
p & =0.400\end{aligned}$ & $\begin{aligned} r & =-0.04 \\
p & =0.889\end{aligned}$ & $\begin{array}{c}r=0.09 \\
p=0.767\end{array}$ & $\begin{array}{c}r=0.07 \\
p=0.831\end{array}$ & $\begin{array}{c}r=0.21 \\
p=0.494\end{array}$ & $\begin{array}{c}r=0.04 \\
p=0.887\end{array}$ & $\begin{aligned} r & =-0.26 \\
p & =0.305\end{aligned}$ \\
\hline LDL-C (mg/dl) & $\begin{aligned} r & =-0.16 \\
p & =0.708\end{aligned}$ & $\begin{array}{l}r=-0.14 \\
p=0.740\end{array}$ & $\begin{array}{c}r=0.13 \\
p=0.754\end{array}$ & $\begin{aligned} r & =-0.03 \\
p & =0.937\end{aligned}$ & $\begin{array}{c}r=0.10 \\
p=0.806\end{array}$ & $\begin{array}{c}r=0.12 \\
p=0.777\end{array}$ & $\begin{array}{c}r=0.26 \\
p=0.411\end{array}$ \\
\hline HDL-C (mg/dl) & $\begin{array}{c}r=0.42 \\
p=0.156\end{array}$ & $\begin{array}{c}r=0.12 \\
p=0.696\end{array}$ & $\begin{aligned} r & =-0.06 \\
p & =0.840\end{aligned}$ & $\begin{aligned} r & =0.09 \\
p & =0.775\end{aligned}$ & $\begin{aligned} r & =-0.20 \\
p & =0.504\end{aligned}$ & $\begin{aligned} r & =-0.04 \\
p & =0.888\end{aligned}$ & $\begin{array}{c}r=0.09 \\
p=0.736\end{array}$ \\
\hline hsCRP (mg/l) & $\begin{array}{c}r=0.33 \\
p=0.268\end{array}$ & $\begin{array}{c}r=0.36 \\
p=0.225\end{array}$ & $\begin{array}{l}r=-0.20 \\
p=0.510\end{array}$ & $\begin{array}{l}r=-0.03 \\
p=0.925\end{array}$ & $\begin{aligned} r & =-0.06 \\
p & =0.840\end{aligned}$ & $\begin{aligned} r & =-0.03 \\
p & =0.924\end{aligned}$ & $\begin{aligned} r & =-0.15 \\
p & =0.576\end{aligned}$ \\
\hline $\begin{array}{l}\text { Fibrinogen }(\mathrm{mg} / \\
\mathrm{dl})\end{array}$ & $\begin{array}{c}r=0.13 \\
p=0.677\end{array}$ & $\begin{array}{c}r=0.26 \\
p=0.384\end{array}$ & $\begin{array}{l}r=-0.46 \\
p=0.112\end{array}$ & $\begin{aligned} r & =-0.30 \\
p & =0.311\end{aligned}$ & $\begin{aligned} r & =-0.37 \\
p & =0.208\end{aligned}$ & $\begin{array}{l}r=-0.30 \\
p=0.327\end{array}$ & $\begin{array}{l}r=-0.16 \\
p=0.550\end{array}$ \\
\hline WBC $\left(10^{3} / \mu \mathrm{l}\right)$ & $\begin{array}{c}r=0.16 \\
p=0.578\end{array}$ & $\begin{array}{c}r=0.28 \\
p=0.326\end{array}$ & $\begin{array}{c}r=0.35 \\
p=0.217\end{array}$ & $\begin{aligned} r & =-0.06 \\
p & =0.836\end{aligned}$ & $\begin{aligned} r & =0.17 \\
p & =0.568\end{aligned}$ & $\begin{array}{l}r=-0.21 \\
p=0.472\end{array}$ & $\begin{aligned} r & =-0.08 \\
p & =0.764\end{aligned}$ \\
\hline NEUT $\left(10^{3} / \mu \mathrm{l}\right)$ & $\begin{array}{c}r=0.07 \\
p=0.810\end{array}$ & $\begin{array}{c}r=0.22 \\
p=0.450\end{array}$ & $\begin{array}{c}r=0.42 \\
p=0.133\end{array}$ & $\begin{aligned} r & =-0.07 \\
p & =0.825\end{aligned}$ & $\begin{array}{c}r=0.27 \\
p=0.342\end{array}$ & $\begin{array}{l}r=-0.12 \\
p=0.675\end{array}$ & $\begin{array}{c}r=0.30 \\
p=0.294\end{array}$ \\
\hline LYMPH $\left(10^{3} / \mu \mathrm{l}\right)$ & $\begin{aligned} r & =0.30 \\
p & =0.302\end{aligned}$ & $\begin{array}{c}r=0.25 \\
p=0.381\end{array}$ & $\begin{array}{c}r=0.19 \\
p=0.514\end{array}$ & $\begin{array}{c}r=0.07 \\
p=0.825\end{array}$ & $\begin{aligned} r & =-0.02 \\
p & =0.935\end{aligned}$ & $\begin{aligned} r & =-0.17 \\
p & =0.572\end{aligned}$ & $\begin{array}{c}r=0.04 \\
p=0.886\end{array}$ \\
\hline $\operatorname{MONO}\left(10^{3} / \mu \mathrm{l}\right)$ & $\begin{array}{c}r=0.22 \\
p=0.453\end{array}$ & $\begin{array}{c}r=0.31 \\
p=0.278\end{array}$ & $\begin{aligned} r & =-0.03 \\
p & =0.911\end{aligned}$ & $\begin{aligned} r & =-0.08 \\
p & =0.777\end{aligned}$ & $\begin{aligned} r & =-0.13 \\
p & =0.654\end{aligned}$ & $\begin{array}{l}r=-0.31 \\
p=0.279\end{array}$ & $\begin{array}{l}r=-0.27 \\
p=0.359\end{array}$ \\
\hline $\operatorname{PLT}\left(10^{3} / \mu \mathrm{l}\right)$ & $\begin{aligned} r & =-0.27 \\
p & =0.354\end{aligned}$ & $\begin{array}{c}r=0.08 \\
p=0.789\end{array}$ & $\begin{aligned} r & =-0.04 \\
p & =0.886\end{aligned}$ & $\begin{aligned} r & =-0.28 \\
p & =0.331\end{aligned}$ & $\begin{aligned} r & =-0.01 \\
p & =0.980\end{aligned}$ & $\begin{array}{l}r=-0.13 \\
p=0.666\end{array}$ & $\begin{array}{c}r=0.28 \\
p=0.262\end{array}$ \\
\hline MPV (fl) & $\begin{array}{c}r=0.33 \\
p=0.246\end{array}$ & $\begin{aligned} r & =-0.05 \\
p & =0.867\end{aligned}$ & $\begin{array}{c}r=0.44 \\
p=0.115\end{array}$ & $\begin{array}{c}r=0.72 \\
p=0.003^{*}\end{array}$ & $\begin{array}{c}r=0.51 \\
p=0.062\end{array}$ & $\begin{array}{c}r=0.62 \\
p=0.019^{*}\end{array}$ & $\begin{array}{l}r=0.015 \\
p=0.952\end{array}$ \\
\hline
\end{tabular}

TC-total cholesterol, TG-triglycerides, LDL-C - low-density lipoprotein, HDL-C-high-density lipoprotein, hsCRP-high-sensitivity C-reactive protein, WBC-white blood cells, NEUT - neutrophils, LYMPH - lymphocytes, MONO-monocytes, PLT - platelets, MPV-mean platelet volume, ${ }^{*}$ statistical significance

ward trend in API was observed. However, between the second and the third visit, the other periodontal parameters deteriorated. This proves unsatisfactory cooperation from patients. Only 21 subjects came for the third visit, which means that more than $30 \%$ of patients declined further treatment. In the case of poor cooperation on the part of patients and inability to obtain satisfactory bacterial plaque control, palliative periodontal treatment should be implemented, with particular attention to remotivation in the field of oral hygiene and chemical control of the supragingival plaque.

Biochemical parameters of blood with high diagnostic value in the context of periodontal medicine can be divided into mediators of lipid (TC, TG, LDL-C, HDL-C) and glucose (HbA1c) metabolism, mediators of inflammation (hsCRP, IL-6, TNF- $\alpha$ ) and markers of thrombosis (fibrinogen). A certain role is also attributed to the number of immunologically competent cells: leukocytes [26], neutrophils [27], lymphocytes [27, 28] and monocytes [28]. Most of these parameters were evaluated in our own study. We observed a statistically significant decrease in blood hsCRP concentration and a decrease in the number of WBC and neutrophils between the first and the second visit. At the same time, improvements in plaque control (decrease of PI, API), reduction of PPD and percentage of active periodontal pockets as well as CAL gain were observed. Similarly, Li et al. [28] demonstrated that treatment of periodontitis leads to a statistically significant reduction in the number of CD34(+) cells in the blood, whereas Bokhari et al. [26] observed a significant decrease in WBC. However, in our research, 6 months after SRP, the mean platelet volume (MPV) increased with respect to the value obtained 1 month after treatment, in parallel with deterioration of periodontal treatment outcomes (increased PI, BoP, PPD and CAL loss). In this study, the authors also assessed the relationship between changes in the level of inflammatory mediators and the number of morphotic blood elements on one hand, and changes in the assessed indicators and periodontal parameters on the other hand. The relationships were evaluated in the logistic regression model considering classic CVD risk factors (to exclude their distorting effect). Multivariate analysis showed that the relationships between: 1) change in LDL-C concentration and change in API value; 2) change in the num- 
Table 6. Multivariate analysis showing correlations between changes in biochemical parameters and dental status

\begin{tabular}{|c|c|c|c|c|c|c|}
\hline \multirow{2}{*}{$\begin{array}{l}\text { Dependent } \\
\text { variables in } \\
\text { distinct models }\end{array}$} & \multicolumn{3}{|c|}{ Model I } & \multicolumn{3}{|c|}{ Model II } \\
\hline & $\begin{array}{c}\text { Independent } \\
\text { variables }\end{array}$ & $\begin{array}{c}\text { Standardized } \\
\text { coefficient } b \\
\text { (for dental } \\
\text { parameter only) }\end{array}$ & $\begin{array}{c}p \\
\text { (for dental } \\
\text { parameter } \\
\text { only) } \\
\end{array}$ & $\begin{array}{c}\text { Independent } \\
\text { variables }\end{array}$ & $\begin{array}{c}\text { Standardized } \\
\text { coefficient } b \\
\text { (for dental parameter } \\
\text { only) } \\
\end{array}$ & $\begin{array}{c}p \\
\text { (for dental } \\
\text { parameter only) }\end{array}$ \\
\hline $\begin{array}{l}\text { HDL-C }(\mathrm{mg} / \mathrm{dl}) \\
\text { change from } \\
\text { baseline to } 1 \text { month }\end{array}$ & $\begin{array}{c}\text { age } \\
\text { gender } \\
\text { PPD }(\mathrm{mm})\end{array}$ & 0.518 & $0.007^{*}$ & \multirow{6}{*}{$\begin{array}{l}\text { As in Model I } \\
\text { + tobacco } \\
\text { smoking, } \\
\text { arterial } \\
\text { hypertension, } \\
\text { diabetes } \\
\text { mellitus, BMI, } \\
\text { atherosclerosis } \\
\text { in the family, } \\
\text { income and } \\
\text { education }\end{array}$} & 0.413 & 0.050 \\
\hline $\begin{array}{l}\text { HDL-C }(\mathrm{mg} / \mathrm{dl}) \\
\text { change from } \\
\text { baseline to } 1 \text { month }\end{array}$ & $\begin{array}{c}\text { age } \\
\text { gender } \\
\text { bPP }(\%)\end{array}$ & 0.295 & 0.171 & & 0.257 & 0.252 \\
\hline $\begin{array}{l}\text { LDL-C }(\mathrm{mg} / \mathrm{dl}) \\
\text { change from } \\
\text { baseline to } 1 \text { month }\end{array}$ & $\begin{array}{c}\text { age } \\
\text { gender } \\
\text { API }(\%)\end{array}$ & -0.509 & $0.047^{*}$ & & -0.546 & $0.005^{*}$ \\
\hline $\begin{array}{l}\text { MONO }\left(10^{3} / \mu \mathrm{l}\right) \\
\text { change from } \\
\text { baseline to } 1 \text { month }\end{array}$ & $\begin{array}{l}\text { age } \\
\text { gender } \\
\text { PI }(\%) \\
\end{array}$ & 0.526 & $0.006^{*}$ & & 0.616 & $0010^{*}$ \\
\hline $\begin{array}{l}\text { MPV (fl) change } \\
\text { from } 1 \text { month to } \\
6 \text { months }\end{array}$ & $\begin{array}{c}\text { age } \\
\text { gender } \\
\text { PPD }(\mathrm{mm})\end{array}$ & 0.742 & $0.031^{*}$ & & 0.567 & $0.018^{*}$ \\
\hline $\begin{array}{l}\text { MPV (fl) at } \\
6 \text { months }\end{array}$ & $\begin{array}{l}\text { age } \\
\text { gender } \\
\text { CAL }\end{array}$ & 0.588 & 0.143 & & 0.378 & 0.111 \\
\hline
\end{tabular}

$\overline{H D L-C-h i g h-d e n s i t y ~ l i p o p r o t e i n, ~ P P D ~-~ p r o b i n g ~ p o c k e t ~ d e p t h, ~ b P P ~-~ b l e e d i n g ~ p e r i o d o n t a l ~ p o c k e t s, ~ A P I-a p p r o x i m a l ~ p l a q u e ~ i n d e x, ~ P I-p l a q u e ~ i n d e x, ~}$ CAL-clinical attachment level, ${ }^{*}$ statistical significance

ber of monocytes and change in PI values; 3 ) change of MPV and change of PPD are independent of CVD risk factors. It is difficult to explain the association between the decrease in LDL-C and the increase in API, but it is possible that the reduction in LDL-C was caused by the patient's medication, as well as diet or lifestyle changes as elements of secondary prevention of secondary MI. It is even more likely because the changes took place within 4 months after MI, when the patients were under constant care of a cardiologist. The increase in PI correlated with the number of monocytes in blood, and the increase in PPD correlated with increased MPV. Both the number of monocytes and MPV are indicators of inflammation and may partly explain the effect of periodontitis on cardiovascular risk. Wang et al. [29] pointed to MPV as a marker of periodontitis activity. They reported a positive correlation between increase in MPV and decrease of PPD after periodontal treatment $(r=0.377 ; p=0.014)$. Recently, neutrophils have been indicated as a link between periodontitis and MI $[21,30]$. One study showed that increased serum level of neutrophil markers, myeloperoxidase (MPO) and high matrix metalloproteinase (MMP)-8/tissue inhibitor of metalloproteinase (TIMP)-1 ratio increased the risk of recurrent acute coronary syndrome. Perhaps, if better and more stable effects of periodontal treatment had been obtained in our study, this would have had a greater impact on a reduction in the systemic inflammatory response.
Teeuw et al. [11] conducted a meta-analysis of the influence of periodontal treatment on the biomarker profile of atherosclerosis, on the number of immunologically competent cells and on improvement of vascular endothelial function. Weighted mean difference (WMD) was significant for TC $(-0.11 \mathrm{mmol} / 1,95 \% \mathrm{CI}:-0.21$; $-0.01)$, HDL-C (0.04 mmol/l, 95\% CI: 0.03; 0.06), hsCRP $(-0.50 \mathrm{mg} / \mathrm{l}, 95 \% \mathrm{CI}:-0.78 ;-0.22)$ and for fibrinogen $(-0.47 \mathrm{~g} / 1,95 \% \mathrm{CI}:-0.76 ;-0.17)$ for patients undergoing periodontal treatment, compared to untreated subjects. In addition, statistically significant WMD was observed with respect to concentration of Il- 6 and TNF- $\alpha$. Periodontal treatment improved vascular endothelial function [31]. Interestingly, in patients with a systemic burden (such as CVD, diabetes) changes in the biomarker profile of atherosclerosis and reduction of inflammatory mediators were even more beneficial. WMD for TC was $-0.15 \mathrm{mmol} / \mathrm{l}$ (95\% CI: $-0.29 ;-0.01)$, for TG $-0.24 \mathrm{mmol} / \mathrm{l}$ (95\% CI: -0.26 ; -0.22 ), for HDL-C $0.05 \mathrm{mmol} / \mathrm{l}$ (95\% CI: 0.03 ; $0.06)$ and for hsCRP $-0.71 \mathrm{mg} / \mathrm{l}(95 \% \mathrm{CI}:-1.05 ;-0.36)$. This last issue requires more attention. hsCRP is an acute phase protein, strongly associated with an increased risk of ischemic heart disease, ischemic stroke and vascular mortality [32]. Similarly, lipid profile disorders (increased TC, TG, LDL-C and decreased HDL-C) show strong correlations with the occurrence of CVD [33]. In the cited meta-analysis, periodontal treatment in patients with normal 
body weight, nonsmokers with CVD or diabetes resulted in a statistically significant decrease in blood hsCRP concentration compared to patients without CVD or diabetes, overweight and smokers ( $\triangle \mathrm{CRP}:-0.09 \mathrm{mg} / \mathrm{l}, \mathrm{CI}:-0.60$; $0.42, p=0.73$ ). Similar associations were observed in relation to lipid metabolism mediators. Most likely, this was associated with higher baseline levels of hsCRP, TG, TC and LDL-C in patients with CVD or diabetes, compared to patients without systemic diseases. Overweight, obesity and smoking are the main risk factors for CVD [34]. In addition, overweight and obesity have strong correlations with blood hsCRP levels, and weight reduction results in a decrease of hsCRP concentrations [35]. This may indicate that overweight has a direct effect on hsCRP levels in blood, and in the case of periodontal treatment it masks the effect of this treatment on concentration of inflammatory mediators. On the other hand, the lack of significant changes in atherosclerosis biomarker profile may be related to the direct negative effect of both overweight and tobacco smoking on periodontal tissues. Scientific research has shown that both variables worsen the effects of periodontal treatment [36]. Our study revealed that the prevalence of smoking and overweight/obesity was very high, which may partially explain the obtained results. The cited meta-analysis did not include studies assessing the effect of periodontal treatment on the number of immunologically competent cells, due to the small number of studies published in this field, which additionally underlines the value of our work.

Most clinical trials evaluated the secondary endpoints of the effects of periodontal treatment on cardiovascular risk only in a short period of time. The Periodontitis and Vascular Events Study (PAVE) was a randomized clinical trial that analyzed the effect of conventional treatment of chronic periodontitis (SRP) in 303 subjects as a secondary prophylactic regimen (CVD) over 25 months [37, 38]. Although the incidence of cardiovascular adverse events in the study and control groups was similar $(p=0.85)$, SRP significantly decreased the concentration of hsCRP in blood [the adjusted odds ratio for hsCRP levels $>3 \mathrm{mg} / \mathrm{l}$ at 6 months after treatment versus no treatment was 0.26 (95\% CI: 0.09 to 0.72 )], but this effect was not observed in obese patients.

Our research has several limitations that require attention when interpreting the presented data. First, only MI patients were included in the study. The lack of a control group makes it impossible to compare the results of periodontal treatment, and the impact of this treatment on cardiovascular risk in relation to people without a CVD burden. In addition, all subjects were taking anti-coagulant drugs that could affect the BoP values. Measurements of CAL and PPD were performed at four rather than six measuring points, which could have led to an underestimation of the occurrence of periodontitis. Moreover, bias protection was a critical issue. To curb bias, the investigator who carried out periodontal examinations did not take part in the active treatment, However, due to high drop-out rate (30\%), the risk of bias is high. Some patients could not be reached (changes in phone number) or refused further participation in the study. Despite the standard treatment protocol of periodontitis, the obtained outcomes were not satisfactory. Between the second and the third visit, a deterioration in all assessed periodontal parameters was observed. This indicates that patients after MI require implementation of more stringent treatment regimens, with a greater focus on oral hygiene instructions and a more frequent schedule of follow-up visits, which should be taken into account in future research. Also, the use of blind trial methodology by the observer would be preferable. Nevertheless, the researcher assessing the state of periodontal tissues was calibrated. Much attention should also be paid to the errors related to the influence of interfering variables and modifying factors on the obtained treatment outcomes, but there have been no studies assessing the relationship between periodontitis and MI in which all potential interfering variables were fully controlled. In our study, potential confounding variables were highlighted and an attempt was made to construct statistical models in logistic regression analysis, controlling the most relevant of these parameters.

Summing up, it can be concluded that there is a need for randomized clinical trials to assess the effect of treatment of periodontal disease as a preventive factor (primary and secondary) of acute coronary syndromes. In addition to treatment including mechanotherapy, the importance of using immunomodulatory medications in periodontal treatment should be verified. In the long term, these studies should assess both primary endpoints (CVD death, angina pectoris, MI, stroke) and secondary end points (modifiable CVD risk factors: blood pressure, lipid profile, blood levels of inflammatory markers: hsCRP; parameters assessing the work of myocardium: ejection fraction). Considering the essence of the research topic, it still remains valid.

\section{Conclusions}

The obtained results indicate a relationship between the state of periodontal tissues and mediators of atherosclerosis (hsCRP, LDL-C, HDL-C), the number of immunologically competent cells (WBC, neutrophils, monocytes) and MPV. Patients after MI should be referred to dentists, and preferably to periodontists, to undergo early diagnosis and periodontal treatment [39]. In this group of patients, the treatment program should place a very high emphasis on education in oral health in the context of general health, and often repeat instructions on maintaining optimal oral hygiene. Control visits should be frequent, and breaks between them should not exceed 2-3 months. Obtaining good effects of periodontal treatment may translate into improvement in the field of mediators and markers of in- 
flammation and lipid profile, thus affecting the reduction of cardiovascular risk.

The authors declare no conflict of interest.

\section{References}

1. Nabel EG (2003): Cardiovascular disease. New Engl J Med 349: $60-72$.

2. WHO (2007): Prevention of cardiovascular disease: guidelines for assessment and management of total cardiovascular risk. WHO Press, Geneva.

3. Yusuf S, Hawken S, Ounpuu S, et al. (2004): Effect of potentially modifiable risk factors associated with myocardial infarction in 52 countries (the INTERHEART study): case-control study. Lancet 364: 937-952.

4. Schenkein HA, Loos BG (2013): Inflammatory mechanisms linking periodontal diseases to cardiovascular diseases. J Periodontol 84 (Suppl 4): 51-69.

5. Albandar JM (2011): Underestimation of periodontitis in NHANES surveys. J Periodontol 82: 337-341.

6. Konopka T, Dembowska E, Pietruska M, et al. (2015): Periodontal status and selected parameters of oral condition of poles aged from 65 to 74 years. Przegl Epidemiol 69: 537-542.

7. Lockhart PB, Bolger AF, Papapanou PN, et al. (2012): Periodontal disease and atherosclerotic vascular disease does the evidence support an independent association?: a scientific statement from the American Heart Association. Circulation 125: $2520-2544$.

8. Humphrey LL, Fu R, Buckley DI, et al. (2008): Periodontal disease and coronary heart disease incidence: a systematic review and meta-analysis. J Gen Int Med 23: 2079-2086.

9. Janket SJ, Baird AE, Chuang SK, et al. (2003): Meta-analysis of periodontal disease and risk of coronary heart disease and stroke. Oral Surg Oral Med Oral Pathol Oral Radiol Endod 95: 559-569.

10. Dietrich T, Webb I, Stenhouse L, et al. (2017): Evidence summary: the relationship between oral and cardiovascular disease. Br Dent J 222: 381-385.

11. Teeuw WJ, Slot DE, Susanto H, et al. (2014): Treatment of periodontitis improves the atherosclerotic profile: a systematic review and meta-analysis. J Clin Periodontol 41: 70-79.

12. Nesse W, Abbas F, van der Ploeg I, et al. (2008): Periodontal inflamed surface area: quantifying inflammatory burden. J Clin Periodontol 35: 668-673.

13. Reyes L, Herrera D, Kozarov E, et al. (2013): Periodontal bacterial invasion and infection: contribution to atherosclerotic pathology. J Periodontol 84 (Suppl 4): 30-50.

14. Nakano K, Wada K, Nomura R, et al. (2011): Characterization of aortic aneurysms in cardiovascular disease patients harboring Porphyromonas gingivalis. Oral Dis 17: 370-378.

15. Hayashi C, Gudino CV, Gibson FC $3^{\text {rd }}$, Genco CA (2010): Review: Pathogen-induced inflammation at sites distant from oral infection: bacteria persistence and induction of cell-specific innate immune inflammatory pathways. Mol Oral Microbiol 25: 305-316.

16. Górski B, Nargiełło E, Opolski G, et al. (2016): The association between dental status and systemic lipid profile and inflammatory mediators in patients after myocardial infarction. Adv Clin Exp Med 15: 625-630.
17. Slots J (2013): Periodontology: past, present, perspectives. Periodontol 2000 62: 7-19.

18. O'Leary TJ, Drake RB, Naylor JE (1972): The plaque control record. J Periodontol 43: 38-46.

19. Lange DE, Plegmann HC, Eenboom A, Promsberger A (1977): Klinische bewertungsverfahren zur objektivierung der mundhygiene. Dtsch Zahnarztl Z 32: 44-47

20. Ainamo J, Bay I (1975): Problems and proposals for recording gingivitis and plaque. Int Dent J 25: 229-235.

21. Ndrepepa G, Braun S, Tad T, et al. (2014): Comparative prognostic value of C-reactive protein \& fibrinogen in patients with coronary artery disease. Indian J Med Res 140: 392-400.

22. Górska R, Dembowska E, Konopka TP, et al. (2017): Correlation between the state of periodontal tissues and selected risk factors for periodontitis and myocardial infarction. Adv Clin Exp Med 26: 505-514.

23. Kleber B-M (1999): Anspruch an die initiale parodontale Therapie. Dtsch Zahnärztl Z 54: 218-224.

24. Worthington HV, Clarkson JE, Bryan G, Beirne PV (2013): Routine scale and polish for periodontal health in adults. Cochrane Database Syst Rev 11. DOI: 10.1002/14651858. CD004625.pub4

25. Konopka T, Karolewska E, Rzeszut A (2012): Clinical and microbiological effects of full-mouth disinfection versus conventional treatment of chronic periodonttis. J Stomat 65: 6-23.

26. Bokhari SAH, Khan AA, Butt AK, et al. (2012):. Non-surgical periodontal therapy reduces coronary heart disease risk markers: a randomized controlled trial. J Clin Periodontol 39: 1065-1074.

27. Taylor B, Toffer G, Morel-Kopp MC, et al. (2010):The effect of initial treatment of periodontitis on cardiovascular risk: a randomized controlled trial. Eur J Oral Sci 118: 350-356.

28. Li X, Tse HF, Yiu KH, et al. (2011): Effect of periodontal treatment on circulating CD34(+) cells and peripheral vascular endothelial function: a randomized controlled trial. J Clin Periodontol 38: 148-156.

29. Wang X, Meng H, Xu L, et al. (2015): Mean platelet volume as an inflammatory marker in patients with severe periodontitis. Platelets. 26: 67-71.

30. Marfil-Álvarez R, Mesa F, Arrebola-Moreno A, et al. (2014): Acute myocardial infarct size is related to periodontitis extent and severity. J Dent Res 93: 993-998.

31. Highashi Y, Goto C, Hidaka T, et al. (2009): Oral infecton-inflammatory pathway, periodontitis, is a risk factor for endothelial dysfunction in patients with coronary artery disease. Atherosclerosis 206: 604-610.

32. Kaptoge S, Di Angelantonio E, Lowe G, et al. (2010): C-reactive protein concentration and risk of coronary heart disease, stroke, and mortality: an individual participant metanalysis. Lancet 375: 132-140.

33. Di Angelantonio E, Sarwar N, Perry P, et al. (2009): Major lipids, apolipoproteins, and risk of vascular disease. JAMA 302: 1993-2000.

34. Perk J, De Backer G, Gohlke H, et al. (2012): European Guidelines on cardiovascular disease prevention in clinical practice (version 2012). The Fifth Joint Task Force of the European Society of Cardiology and Other Societies on Cardiovascular Disease Prevention in Clinical Practice (constituted by representatives of nine societies and by invited experts). Eur Heart J 33: 1635-1701.

35. Saleh MH, Bertolami MC, Assef JE, et al. (2012): Improvement of atherosclerotic markers in non-diabetic patients after bariatric surgery. Obes Surg 22: 1701-1707. 
36. Faveri M, Rebello A, Dias RO, et al. (2014): Clinical and micobiological effects of adjunctive metronidazole plus amoxicillin in the treatment of generalized chronic periodontitis: smokers versus non-smokers. J Periodontol 85: 581-591.

37. Beck JD, Couper DJ, Falkner KL, et al. (2008): The Periodontitis and Vascular Events (PAVE) pilot study: adverse events. J Periodontol 79: 90-96.

38. Offenbacher S, Beck JD, Moss K, et al. (2009): Results from the periodontitis and vascular events (PAVE) study: a pilot multicentered, randomized, controlled trial to study effects of periodontal therapy in a secondary prevention model of cardiovascular disease. J Periodontol 80: 190-201.

39. Tonetti MS, Van Dyke TE (2013): Periodontitis and atherosclerotic cardiovascular disease: consensus report of the Joint EFP/AAP Workshop on Periodontitis ans Systemic Diseases. J Clin Periodontol 40 (Suppl 14): 24-29. 